\title{
Spatial variations of drinking water quality monitoring in water treatment plant using environmetric techniques
}

\begin{abstract}
This research investigates the relationship between the physicochemical levels and the drinking water quality in Kuala Kubu Bharu, Selangor, Malaysia based on three different classes of drinking water. The environmetric techniques such as the discriminant analysis (DA), the principal component analysis (PCA) and the factor analysis (FA) were applied to analyze the spatial variation of the most significant physicochemical parameters of the drinking water quality and to determine the source of pollution. Seven physicochemical variables were analyzed. The forward and backward stepwise DA managed to discriminate six and two variables, respectively from the original seven variables. PCA and FA (varimax functionality) were to identify the origin of each water quality variable based on the three different drinking water classes. This study shows that environmetric method is the ideal way into provide meaningful information on the spatial variability of sophisticated drinking water quality data.
\end{abstract}

Keyword: Environmetric techniques; Discriminant analysis; Principal component analysis; Factor analysis; Drinking water quality 